\title{
STABILITY OF HOMOMORPHISMS AND DERIVATIONS IN PROPER JCQ*-TRIPLES ASSOCIATED TO THE PEXIDERIZED CAUCHY TYPE MAPPING
}

\author{
Abbas Najati, G. Zamani Eskandani, and Choonkil Park
}

\begin{abstract}
In this paper, we investigate homomorphisms in proper $J C Q^{*}$-triples and derivations on proper $J C Q^{*}$-triples associated to the following Pexiderized functional equation

$$
f(x+y+z)=f_{0}(x)+f_{1}(y)+f_{2}(z) .
$$

This is applied to investigate homomorphisms and derivations in proper $J C Q^{*}$-triples.
\end{abstract}

\section{Introduction and preliminaries}

Ulam [14] gave a talk before the Mathematics Club of the University of Wisconsin in which he discussed a number of unsolved problems. Among these was the following question concerning the stability of homomorphisms.

Let $\left(G_{1}, *\right)$ be a group and let $\left(G_{2}, \diamond, d\right)$ be a metric group with the metric $d(\cdot, \cdot)$. Given $\epsilon>0$, does there exist a $\delta(\epsilon)>0$ such that if a mapping $h: G_{1} \rightarrow$ $G_{2}$ satisfies the inequality

$$
d(h(x * y), h(x) \diamond h(y))<\delta
$$

for all $x, y \in G_{1}$, then there is a homomorphism $H: G_{1} \rightarrow G_{2}$ with

$$
d(h(x), H(x))<\epsilon
$$

for all $x \in G_{1}$ ?

Hyers [7] considered the case of approximately additive mappings $f: E \rightarrow$ $E^{\prime}$, where $E$ and $E^{\prime}$ are Banach spaces and $f$ satisfies Hyers inequality

$$
\|f(x+y)-f(x)-f(y)\| \leq \epsilon
$$

for all $x, y \in E$. It was shown that the limit

$$
L(x)=\lim _{n \rightarrow \infty} \frac{f\left(2^{n} x\right)}{2^{n}}
$$

Received February 9, 2008.

2000 Mathematics Subject Classification. Primary 39B52, 47N50, 47L60, 47L90, 46H35, $46 \mathrm{~B} 03$.

Key words and phrases. generalized Hyers-Ulam stability, proper $J C Q^{*}$-triples homomorphism, proper $J C Q^{*}$-triples derivation. 
exists for all $x \in E$ and that $L: E \rightarrow E^{\prime}$ is the unique additive mapping satisfying

$$
\|f(x)-L(x)\| \leq \epsilon .
$$

Th. M. Rassias [13] provided a generalization of Hyers' theorem which allows the Cauchy difference to be unbounded.

We recall some basic facts concerning quasi *-algebras.

Definition 1.1. Let $A$ be a linear space and $A_{0}$ be a $*$-algebra contained in $A$ as a subspace. We say that $A$ is a quasi *-algebra over $A_{0}$ if

(i) the right and left multiplications of an element of $A$ and an element of $A_{0}$ are always defined and linear;

(ii) $x_{1}\left(x_{2} a\right)=\left(x_{1} x_{2}\right) a,\left(a x_{1}\right) x_{2}=a\left(x_{1} x_{2}\right)$ and $x_{1}\left(a x_{2}\right)=\left(x_{1} a\right) x_{2}$ for all $x_{1}, x_{2} \in A_{0}$ and all $a \in A$;

(iii) an involution *, which extends the involution of $A_{0}$, is defined in $A$ with the property $(a b)^{*}=b^{*} a^{*}$, whenever the multiplication is defined.

Quasi *-algebras $[8,9]$ arise in natural way as completions of locally convex *-algebras whose multiplication is not jointly continuous; in this case one has to deal with topological quasi $*$-algebras.

A quasi *-algebra $\left(A, A_{0}\right)$ is called topological if a locally convex topology $\tau$ on $A$ is given such that:

(i) the involution $a \mapsto a^{*}$ is continuous for each $a \in A$,

(ii) the mappings $a \mapsto a b$ and $a \mapsto b a$ are continuous for each $a \in A$ and $b \in A_{0}$,

(iii) $A_{0}$ is dense in $A[\tau]$.

Throughout this paper, we suppose that a locally convex quasi $*$-algebra $\left(A, A_{0}\right)$ is complete. For an overview on partial *-algebra and related topics we refer to [1].

In a series of papers [2], [3], [4], [5] many authors have considered a special class of quasi *-algebras, called proper $C Q^{*}$-algebras, which arise as completions of $C^{*}$-algebras. They can be introduced in the following way:

Definition 1.2. Let $A$ be a Banach module over the $C^{*}$-algebra $A_{0}$ with involution $*$ and $C^{*}$-norm $\|\cdot\|_{0}$ such that $A_{0} \subset A$. We say that $\left(A, A_{0}\right)$ is a proper $C Q^{*}$-algebra if

(i) $A_{0}$ is dense in $A$ with respect to its norm $\|\cdot\|$;

(ii) $(a b)^{*}=b^{*} a^{*}$ whenever the multiplication is defined;

(iii) $\|y\|_{0}=\max \left\{\sup _{a \in A,\|a\| \leq 1}\|a y\|, \sup _{a \in A,\|a\| \leq 1}\|y a\|\right\}$ for all $y \in A_{0}$.

A proper $C Q^{*}$-algebra $\left(A, A_{0}\right)$ is said to have a unit $e$ if there exists an element $e \in A_{0}$ such that $a e=e a=a$ for all $a \in A$. In this paper we will always assume that the proper $C Q^{*}$-algebra under consideration have an identity.

Definition 1.3. A proper $C Q^{*}$-algebra $\left(A, A_{0}\right)$, endowed with the Jordan triple product

$$
\{z, x, w\}=\frac{1}{2}\left\{z x^{*} w+w x^{*} z\right\}
$$


for all $x \in A$ and all $z, w \in A_{0}$, is called a proper $J C Q^{*}$-triple, and denoted by $\left(A, A_{0},\{., .,\}.\right)$.

Definition 1.4. Let $\left(A, A_{0},\{., .,\}.\right)$ and $\left(B, B_{0},\{., .,\}.\right)$ be proper $J C Q^{*}$ triples.

(i) A $\mathbb{C}$-linear mapping $H: A \rightarrow B$ is called a proper $J C Q^{*}$-triple homomorphism if $H(z) \in B_{0}$ and $H(\{z, x, w\})=\{H(z), H(x), H(w)\}$ for all $z, w \in A_{0}$ and all $x \in A$.

(ii) A $\mathbb{C}$-linear mapping $\delta: A_{0} \rightarrow A$ is called a proper $J C Q^{*}$-triple derivation if

$$
\begin{aligned}
& \delta\left(\left\{w_{0}, w_{1}, w_{2}\right\}\right)=\left\{\delta\left(w_{0}\right), w_{1}, w_{2}\right\}+\left\{w_{0}, \delta\left(w_{1}\right), w_{2}\right\}+\left\{w_{0}, w_{1}, \delta\left(w_{2}\right)\right\} \\
& \quad \text { for all } w_{0}, w_{1}, w_{2} \in A_{0} .
\end{aligned}
$$

A. Najati and C. Park [10] investigated homomorphisms in quasi-Banach algebras associated to the Pexiderrized Cauchy function equation. C. Park and Th. M. Rassias [12] investigated homomorphisms in proper $J C Q^{*}$-triples and derivations on proper $J C Q^{*}$-triples.

In this paper, we investigate homomorphisms and derivations in proper $J C Q^{*}$-triples associated to the following Pexiderized Cauchy type functional equation

$$
f(x+y+z)=f_{0}(x)+f_{1}(y)+f_{2}(z)
$$

Throughout this paper, assume that $k$ is a fixed positive integer.

\section{Homomorphisms in proper $J C Q^{*}$-triples}

Throughout this section, assume that $\left(A, A_{0},\{., .\},\right)$ is a proper $J C Q^{*}$ triple with $C^{*}$-norm $\|\cdot\|_{A_{0}}$ and norm $\|\cdot\|_{A}$, and that $\left(B, B_{0},\{., .,\}.\right)$ is a proper $J C Q^{*}$-triple with $C^{*}$-norm $\|\cdot\|_{B_{0}}$ and norm $\|\cdot\|_{B}$.

Theorem 2.1. Let $\varphi: A \times A \times A \rightarrow[0,+\infty)$ be a function such that

$$
\lim _{n \rightarrow \infty} \frac{1}{2^{n}} \varphi\left(2^{n} w_{0}, x, w_{2}\right)=0
$$

for all $w_{0}, w_{2} \in A_{0}$ and all $x \in A$. Assume that $f, f_{i}: A \rightarrow B(0 \leq i \leq 2)$ are mappings with $f(0)=0$ and $f(w), f_{0}(0), f_{2}(0) \in B_{0}$ for all $w \in A_{0}$ and

$$
\begin{gathered}
\left\|\mu f(x)-f_{0}(y)-f_{1}(z)-f_{2}(t)\right\|_{B} \leq\left\|k f\left(\frac{\mu x+y+z+t}{k}\right)\right\|_{B}, \\
\left\|f\left(\left\{w_{0}, x, w_{2}\right\}\right)+\left\{f_{0}\left(w_{0}\right), f_{1}(x), f_{2}\left(w_{2}\right)\right\}\right\|_{B} \leq \varphi\left(w_{0}, x, w_{2}\right)
\end{gathered}
$$

for all $\mu \in \mathbb{T}^{1}:=\{\mu \in \mathbb{C}:|\mu|=1\}$, all $w_{0}, w_{2} \in A_{0}$ and all $x, y, z, t \in A$. Then the mapping $f: A \rightarrow B$ is a proper JCQ* $Q^{*}$-triple homomorphism. Moreover,

$$
f(x)=f_{0}(0)-f_{0}(x)=f_{1}(0)-f_{1}(x)=f_{2}(0)-f_{2}(x)
$$

for all $x \in A$. 
Proof. Letting $\mu=1, x=y=z=t=0$ in (2.2), we get

$$
f_{0}(0)+f_{1}(0)+f_{2}(0)=0 \text {. }
$$

So by letting $\mu=1, y=-x$, and $z=t=0$ in (2.2), we get

$$
f(x)=f_{0}(-x)-f_{0}(0)
$$

for all $x \in A$. Similarly, we have

$$
f(x)=f_{1}(-x)-f_{1}(0)=f_{2}(-x)-f_{2}(0)
$$

for all $x \in A$. So $f_{0}(w), f_{2}(w) \in B_{0}$ for all $w \in A_{0}$.

It follows from (2.2) that

$$
\begin{aligned}
& \|\mu f(x+y)-f(\mu x)-f(\mu y)\|_{B} \\
= & \left\|\mu f(x+y)-f_{0}(-\mu x)-f_{1}(-\mu y)-f_{2}(0)\right\|_{B}=0
\end{aligned}
$$

for all $x, y \in A$ and all $\mu \in \mathbb{T}^{1}$. Therefore, the mapping $f: A \rightarrow B$ is additive and $f(\mu x)=\mu f(x)$ for all $x \in A$ and all $\mu \in \mathbb{T}^{1}$. By the same reasoning as in the proof of Theorem 2.1 of [11], the mapping $f: A \rightarrow B$ is $\mathbb{C}$-linear. If at least $w_{0}=0$ or $x=0$ or $w_{2}=0$, then $f\left(\left\{w_{0}, x, w_{2}\right\}\right)=f(0)=0$, and hence by $(2.1)$ and (2.3), we have

$$
\lim _{n \rightarrow \infty} \frac{1}{2^{n}}\left\|\left\{f_{0}\left(2^{n} w_{0}\right), f_{1}(x), f_{2}\left(w_{2}\right)\right\}\right\|_{B}=0 .
$$

Also, we have

$$
f(x)=f_{i}(0)-f_{i}(x)
$$

for all $x \in A$ and all $0 \leq i \leq 2$. So (2.3) implies that

$$
\begin{aligned}
& \left\|f\left(\left\{w_{0}, x, w_{2}\right\}\right)-\left\{f\left(w_{0}\right), f(x), f\left(w_{2}\right)\right\}\right\|_{B} \\
= & \lim _{n \rightarrow \infty} \frac{1}{2^{n}}\left\|f\left(2^{n}\left\{w_{0}, x, w_{2}\right\}\right)-\left\{f\left(2^{n} w_{0}\right), f(x), f\left(w_{2}\right)\right\}\right\|_{B} \\
= & \lim _{n \rightarrow \infty} \frac{1}{2^{n}}\left\|f\left(2^{n}\left\{w_{0}, x, w_{2}\right\}\right)+\left\{f_{0}\left(2^{n} w_{0}\right), f_{1}(x), f_{2}\left(w_{2}\right)\right\}\right\|_{B} \\
\leq & \lim _{n \rightarrow \infty} \frac{1}{2^{n}} \varphi\left(2^{n} w_{0}, x, w_{2}\right)=0
\end{aligned}
$$

for all $w_{0}, w_{2} \in A_{0}$ and all $x \in A$. So

$$
f\left(\left\{w_{0}, x, w_{2}\right\}\right)=\left\{f\left(w_{0}\right), f(x), f\left(w_{2}\right)\right\}
$$

for all $w_{0}, w_{2} \in A_{0}$ and all $x \in A$. Since $f(w) \in B_{0}$ for all $w \in A_{0}$, the mapping $f: A \rightarrow B$ is a proper $J C Q^{*}$-triple homomorphism, as desired.

Remark 2.2. We can formulate a similar theorem if we replace the condition (2.1) by one of the following conditions

- $\lim _{n \rightarrow \infty} \frac{1}{2^{n}} \varphi\left(w_{0}, 2^{n} x, w_{2}\right)=0$;

- $\lim _{n \rightarrow \infty} \frac{1}{2^{n}} \varphi\left(w_{0}, x, 2^{n} w_{2}\right)=0$;

- $\lim _{n \rightarrow \infty} \frac{1}{8^{n}} \varphi\left(2^{n} w_{0}, 2^{n} x, 2^{n} w_{2}\right)=0$

for all $w_{0}, w_{2} \in A_{0}$ and all $x \in A$. 
Remark 2.3. We can formulate a similar theorem if we replace the condition (2.1) by one of the following conditions

- $\lim _{n \rightarrow \infty} 2^{n} \varphi\left(\frac{w_{0}}{2^{n}}, x, w_{2}\right)=0$;

- $\lim _{n \rightarrow \infty} 2^{n} \varphi\left(w_{0}, \frac{x}{2^{n}}, w_{2}\right)=0$;

- $\lim _{n \rightarrow \infty} 2^{n} \varphi\left(w_{0}, x, \frac{w_{2}}{2^{n}}\right)=0$;

- $\lim _{n \rightarrow \infty} 8^{n} \varphi\left(\frac{w_{0}}{2^{n}}, \frac{x}{2^{n}}, \frac{w_{2}}{2^{n}}\right)=0$

for all $w_{0}, w_{2} \in A_{0}$ and all $x \in A$.

Corollary 2.4. Let $\theta, r_{i}(0 \leq i \leq 2)$ be non-negative real numbers such that $r_{0}+r_{1}+r_{2} \neq 3$ or $r_{j} \neq 1$ for some $0 \leq j \leq 2$. Suppose that $f, f_{i}: A \rightarrow B(0 \leq$ $i \leq 2$ ) are mappings satisfying $(2.2)$ with $f(0)=0$ and $f(w), f_{0}(0), f_{2}(0) \in B_{0}$ for all $w \in A_{0}$. Let

$$
\left\|f\left(\left\{w_{0}, x, w_{2}\right\}\right)+\left\{f_{0}\left(w_{0}\right), f_{1}(x), f_{2}\left(w_{2}\right)\right\}\right\|_{B} \leq \theta\left\|w_{0}\right\|_{A}^{r_{0}}\|x\|_{A}^{r_{1}}\left\|w_{2}\right\|_{A}^{r_{2}}
$$

for all $w_{0}, w_{2} \in A_{0}$ and all $x \in A$ (by putting $\|\cdot\|_{A}^{0}=1$ ). Then the mapping $f: A \rightarrow B$ is a proper JCQ $Q^{*}$-triple homomorphism. Moreover,

$$
f(x)=f_{0}(0)-f_{0}(x)=f_{1}(0)-f_{1}(x)=f_{2}(0)-f_{2}(x)
$$

for all $x \in A$.

Proof. It follows from Theorem 2.1 and Remarks 2.2 and 2.3 .

Corollary 2.5. Let $\theta, r_{i}(0 \leq i \leq 2)$ be non-negative real numbers such that $r_{j} \in[0,1)$ for some $0 \leq j \leq 2$ or $r_{i}<3$ (respectively, $r_{i}>3$ ) for all $0 \leq i \leq 2$. Suppose that $f, f_{i}: A \rightarrow B(0 \leq i \leq 2)$ are mappings satisfying (2.2) with $f(0)=0$ and $f(w), f_{0}(0), f_{2}(0) \in B_{0}$ for all $w \in A_{0}$. Let

$$
\left\|f\left(\left\{w_{0}, x, w_{2}\right\}\right)+\left\{f_{0}\left(w_{0}\right), f_{1}(x), f_{2}\left(w_{2}\right)\right\}\right\|_{B} \leq \theta\left(\left\|w_{0}\right\|_{A}^{r_{0}}+\|x\|_{A}^{r_{1}}+\left\|w_{2}\right\|_{A}^{r_{2}}\right)
$$

for all $w_{0}, w_{2} \in A_{0}$ and all $x \in A$ (by putting $\|\cdot\|_{A}^{0}=1$ ). Then the mapping $f: A \rightarrow B$ is a proper JCQ*-triple homomorphism. Moreover,

$$
f(x)=f_{0}(0)-f_{0}(x)=f_{1}(0)-f_{1}(x)=f_{2}(0)-f_{2}(x)
$$

for all $x \in A$.

Proof. The result follows from Theorem 2.1 and Remarks 2.2 and 2.3.

\section{Derivations on proper $J C Q^{*}$-triples}

Throughout this section, assume that $\left(A, A_{0},\{., .,\}.\right)$ is a proper $J C Q^{*}$-triple with $C^{*}$-norm $\|\cdot\|_{A_{0}}$ and norm $\|\cdot\|_{A}$.

We investigate derivations on proper $J C Q^{*}$-triples.

Theorem 3.1. Let $\varphi: A_{0} \times A_{0} \times A_{0} \rightarrow[0,+\infty)$ be a function satisfying (2.1) for all $x, w_{0}, w_{2} \in A_{0}$. Assume that $f, f_{i}: A_{0} \rightarrow A(0 \leq i \leq 2)$ are mappings with $f(0)=0$. Let

$$
\left\|\mu f(x)-f_{0}\left(w_{0}\right)-f_{1}\left(w_{1}\right)-f_{2}\left(w_{2}\right)\right\|_{A} \leq\left\|k f\left(\frac{\mu x+w_{0}+w_{1}+w_{2}}{k}\right)\right\|_{A},
$$




$$
\begin{aligned}
\| f\left(\left\{w_{0}, w_{1}, w_{2}\right\}\right)+\{ & \left.f_{0}\left(w_{0}\right), w_{1}, w_{2}\right\}+\left\{w_{0}, f_{1}\left(w_{1}\right), w_{2}\right\} \\
& +\left\{w_{0}, w_{1}, f_{2}\left(w_{2}\right)\right\} \|_{A} \leq \varphi\left(w_{0}, w_{1}, w_{2}\right)
\end{aligned}
$$

for all $x, w_{0}, w_{1}, w_{2} \in A_{0}$. Then the mapping $f: A_{0} \rightarrow A$ is a proper JCQ*triple derivation. Moreover,

$$
f(x)=f_{0}(0)-f_{0}(x)=f_{1}(0)-f_{1}(x)=f_{2}(0)-f_{2}(x)
$$

for all $x \in A_{0}$.

Proof. By the same reasoning as in the proof of Theorem 2.1, the mapping $f: A_{0} \rightarrow A$ is $\mathbb{C}$-linear and

$$
f(x)=f_{0}(0)-f_{0}(x)=f_{1}(0)-f_{1}(x)=f_{2}(0)-f_{2}(x)
$$

for all $x \in A_{0}$. If at least $w_{0}=0$ or $w_{1}=0$ or $w_{2}=0$, then $f\left(\left\{w_{0}, w_{1}, w_{2}\right\}\right)=$ $f(0)=0$ and by $(2.1)$ and $(3.2)$, we have

$\lim _{n \rightarrow \infty} \frac{1}{2^{n}}\left\|\left\{f_{0}\left(2^{n} w_{0}\right), w_{1}, w_{2}\right\}+\left\{2^{n} w_{0}, f_{1}\left(w_{1}\right), w_{2}\right\}+\left\{2^{n} w_{0}, w_{1}, f_{2}\left(w_{2}\right)\right\}\right\|_{A}=0$.

So (3.2) implies that

$$
\begin{aligned}
& \quad\left\|f\left(\left\{w_{0}, w_{1}, w_{2}\right\}\right)-\left\{f\left(w_{0}\right), w_{1}, w_{2}\right\}-\left\{w_{0}, f\left(w_{1}\right), w_{2}\right\}-\left\{w_{0}, w_{1}, f\left(w_{2}\right)\right\}\right\|_{A} \\
& =\lim _{n \rightarrow \infty} \frac{1}{2^{n}} \| f\left(2^{n}\left\{w_{0}, w_{1}, w_{2}\right\}\right)+\left\{f_{0}\left(2^{n} w_{0}\right), w_{1}, w_{2}\right\}+\left\{2^{n} w_{0}, f_{1}\left(w_{1}\right), w_{2}\right\} \\
& \quad+\left\{2^{n} w_{0}, w_{1}, f_{2}\left(w_{2}\right)\right\} \|_{A} \\
& \leq \lim _{n \rightarrow \infty} \frac{1}{2^{n}} \varphi\left(2^{n} w_{0}, w_{1}, w_{2}\right)=0
\end{aligned}
$$

for all $w_{0}, w_{1}, w_{2} \in A_{0}$. Hence

$$
f\left(\left\{w_{0}, w_{1}, w_{2}\right\}\right)=\left\{f\left(w_{0}\right), w_{1}, w_{2}\right\}+\left\{w_{0}, f\left(w_{1}\right), w_{2}\right\}+\left\{w_{0}, w_{1}, f\left(w_{2}\right)\right\}
$$

for all $w_{0}, w_{1}, w_{2} \in A_{0}$.

Therefore the mapping $f: A_{0} \rightarrow A$ is a proper $J C Q^{*}$-triple derivation.

Remark 3.2. We can formulate a similar theorem if we replace the condition (2.1) by one of the following conditions

- $\lim _{n \rightarrow \infty} \frac{1}{2^{n}} \varphi\left(w_{0}, 2^{n} w_{1}, w_{2}\right)=0$;

- $\lim _{n \rightarrow \infty} \frac{1}{2^{n}} \varphi\left(w_{0}, w_{1}, 2^{n} w_{2}\right)=0$

- $\lim _{n \rightarrow \infty} \frac{1}{8^{n}} \varphi\left(2^{n} w_{0}, 2^{n} w_{1}, 2^{n} w_{2}\right)=0$

for all $w_{0}, w_{1}, w_{2} \in A_{0}$.

Remark 3.3. We can formulate a similar theorem if we replace the condition (2.1) by one of the following conditions

- $\lim _{n \rightarrow \infty} 2^{n} \varphi\left(\frac{w_{0}}{2^{n}}, w_{1}, w_{2}\right)=0$;

- $\lim _{n \rightarrow \infty} 2^{n} \varphi\left(w_{0}, \frac{w_{1}}{2^{n}}, w_{2}\right)=0$;

- $\lim _{n \rightarrow \infty} 2^{n} \varphi\left(w_{0}, w_{1}, \frac{w_{2}}{2^{n}}\right)=0$;

- $\lim _{n \rightarrow \infty} 8^{n} \varphi\left(\frac{w_{0}}{2^{n}}, \frac{w_{1}}{2^{n}}, \frac{w_{2}}{2^{n}}\right)=0$

for all $w_{0}, w_{1}, w_{2} \in A_{0}$. 
Corollary 3.4. Let $\theta, r_{i}(0 \leq i \leq 2)$ be non-negative real numbers such that $r_{0}+r_{1}+r_{2} \neq 3$ or $r_{j} \neq 1$ for some $0 \leq j \leq 2$. Suppose that $f, f_{i}: A_{0} \rightarrow A$ $(0 \leq i \leq 2)$ are mappings satisfying $(3.1)$ with $f(0)=0$. Let

$$
\begin{aligned}
& \| f\left(\left\{w_{0}, w_{1}, w_{2}\right\}\right)+\left\{f_{0}\left(w_{0}\right), w_{1}, w_{2}\right\}+\left\{w_{0}, f_{1}\left(w_{1}\right), w_{2}\right\} \\
& +\left\{w_{0}, w_{1}, f_{2}\left(w_{2}\right)\right\}\left\|_{A} \leq \theta\right\| w_{0}\left\|_{A_{0}}^{r_{0}}\right\| w_{1}\left\|_{A_{0}}^{r_{1}}\right\| w_{2} \|_{A_{0}}^{r_{2}}
\end{aligned}
$$

for all $w_{0}, w_{1}, w_{2} \in A_{0}$ (by putting $\|\cdot\|_{A_{0}}^{0}=1$ ). Then the mapping $f: A_{0} \rightarrow A$ is a proper $J C Q^{*}$-triple derivation. Moreover,

$$
f(x)=f_{0}(0)-f_{0}(x)=f_{1}(0)-f_{1}(x)=f_{2}(0)-f_{2}(x)
$$

for all $x \in A$.

Proof. It follows from Theorem 3.1 and Remarks 3.2 and 3.3.

Corollary 3.5. Let $\theta, r_{i}(0 \leq i \leq 2)$ be non-negative real numbers such that $r_{j} \in[0,1)$ for some $0 \leq j \leq 2$ or $r_{i}<3$ (respectively, $r_{i}>3$ ) for all $0 \leq i \leq 2$. Suppose that $f, f_{i}: A_{0} \rightarrow A(0 \leq i \leq 2)$ are mappings satisfying (3.1) with $f(0)=0$. Let

$$
\begin{aligned}
\| f\left(\left\{w_{0}, w_{1}, w_{2}\right\}\right)+\{ & \left.f_{0}\left(w_{0}\right), w_{1}, w_{2}\right\}+\left\{w_{0}, f_{1}\left(w_{1}\right), w_{2}\right\} \\
& +\left\{w_{0}, w_{1}, f_{2}\left(w_{2}\right)\right\} \|_{A} \leq \theta\left(\left\|w_{0}\right\|_{A_{0}}^{r_{0}}+\left\|w_{1}\right\|_{A_{0}}^{r_{1}}+\left\|w_{2}\right\|_{A_{0}}^{r_{2}}\right)
\end{aligned}
$$

for all $w_{0}, w_{1}, w_{2} \in A_{0}$ (by putting $\|\cdot\|_{A_{0}}^{0}=1$ ). Then the mapping $f: A_{0} \rightarrow A$ is a proper JCQ*-triple derivation. Moreover,

$$
f(x)=f_{0}(0)-f_{0}(x)=f_{1}(0)-f_{1}(x)=f_{2}(0)-f_{2}(x)
$$

for all $x \in A_{0}$.

Proof. It follows from Theorem 3.1 and Remarks 3.2 and 3.3.

\section{Stability of homomorphisms on proper $J C Q^{*}$-triples}

In this section, by using an idea of Găvruta [6], we prove the generalized Hyers-Ulam stability of homomorphisms in proper $J C Q^{*}$-triples.

Theorem 4.1. Let $\varphi: A \times A \times A \rightarrow[0,+\infty)$ be a function such that $\varphi(0,0,0)=$ 0 and

$$
\begin{gathered}
\lim _{n \rightarrow \infty} \frac{1}{2^{n}} \varphi\left(2^{n} x, 2^{n} y, 2^{n} z\right)=0, \\
\widetilde{\varphi}(x):=\sum_{i=0}^{\infty} \frac{1}{2^{i}}\left[\varphi\left(2^{i} x, 2^{i} x, 0\right)+\varphi\left(2^{i} x, 0,0\right)+\varphi\left(0,2^{i} x, 0\right)\right]<\infty
\end{gathered}
$$

for all $x, y, z \in A$. Suppose that $f, f_{i}: A \rightarrow B(0 \leq i \leq 2)$ are mappings satisfying $f(0)=0$ and $f(w), f_{i}(w) \in B_{0}$ for all $w \in A_{0}$ and all $0 \leq i \leq 2$. Let

$$
\begin{gathered}
\left\|f(\mu x+\mu y+\mu z)-\mu f_{0}(x)-\mu f_{1}(y)-\mu f_{2}(z)\right\|_{B} \leq \varphi(x, y, z), \\
\left\|f\left(w_{0}+w_{1}+w_{2}\right)-f_{0}\left(w_{0}\right)-f_{1}\left(w_{1}\right)-f_{2}\left(w_{2}\right)\right\|_{B_{0}} \leq \varphi\left(w_{0}, w_{1}, w_{2}\right),
\end{gathered}
$$




$$
\left\|f\left(\left\{w_{0}, x, w_{1}\right\}\right)-\left\{f_{0}\left(w_{0}\right), f_{1}(x), f_{2}\left(w_{2}\right)\right\}\right\|_{B} \leq \varphi\left(w_{0}, x, w_{2}\right)
$$

for all $\mu \in \mathbb{T}^{1}$, all $w_{0}, w_{1}, w_{2} \in A_{0}$ and all $x, y, z \in A$. Then there exists $a$ unique proper JCQ*-triple homomorphism $H: A \rightarrow B$ such that

$$
\begin{aligned}
\|f(x)-H(x)\|_{B} & \leq \frac{1}{2} \widetilde{\varphi}(x), \\
\left\|f_{0}(x)-f_{0}(0)-H(x)\right\|_{B} & \leq \frac{1}{2} \widetilde{\varphi}(x)+\varphi(x, 0,0), \\
\left\|f_{1}(x)-f_{1}(0)-H(x)\right\|_{B} & \leq \frac{1}{2} \widetilde{\varphi}(x)+\varphi(0, x, 0), \\
\left\|f_{2}(x)-f_{2}(0)-H(x)\right\|_{B} & \leq \frac{1}{2} \widetilde{\varphi}(x)+\varphi(0,0, x)
\end{aligned}
$$

for all $x \in A$.

Proof. Letting $y=z=0$ and $\mu=1$ in (4.3), we get

$$
\left\|f(x)-f_{0}(x)-f_{1}(0)-f_{2}(0)\right\|_{B} \leq \varphi(x, 0,0)
$$

for all $x \in A$. Similarly, we get

$$
\begin{gathered}
\left\|f(y)-f_{1}(y)-f_{0}(0)-f_{2}(0)\right\|_{B} \leq \varphi(0, y, 0), \\
\left\|f(z)-f_{2}(z)-f_{0}(0)-f_{1}(0)\right\|_{B} \leq \varphi(0,0, z)
\end{gathered}
$$

for all $y, z \in A$. Since $f_{0}(0)+f_{1}(0)+f_{2}(0)=0$, by using (4.3), (4.7), (4.8) and (4.9), we get

$$
\|f(\mu x+\mu y+\mu z)-\mu f(x)-\mu f(y)-\mu f(z)\|_{B} \leq \psi(x, y, z),
$$

where

$$
\psi(x, y, z):=\varphi(x, y, z)+\varphi(x, 0,0)+\varphi(0, y, 0)+\varphi(0,0, z)
$$

for all $\mu \in \mathbb{T}^{1}$ and all $x, y, z \in A$. Letting $y=x, z=0$ and $\mu=1$ in (4.10), we get

$$
\|f(2 x)-2 f(x)\|_{B} \leq \psi(x, x, 0)
$$

for all $x \in A$. Replacing $x$ by $2^{n} x$ in (4.11) and dividing both sides of (4.11) by $2^{n+1}$, we get

$$
\left\|\frac{f\left(2^{n+1} x\right)}{2^{n+1}}-\frac{f\left(2^{n} x\right)}{2^{n}}\right\|_{B} \leq \frac{1}{2^{n+1}} \psi\left(2^{n} x, 2^{n} x, 0\right)
$$

for all $x \in A$ and all non-negative integers $n$. By (4.12), we have

$$
\left\|\frac{f\left(2^{n+1} x\right)}{2^{n+1}}-\frac{f\left(2^{m} x\right)}{2^{m}}\right\|_{B} \leq \frac{1}{2} \sum_{i=m}^{n} \frac{\psi\left(2^{i} x, 2^{i} x, 0\right)}{2^{i}}
$$

for all $x \in A$ and all non-negative integers $n$ and $m$ with $n \geq m$. Thus we conclude from (4.2) and (4.13) that the sequence $\left\{\frac{1}{2^{n}} f\left(2^{n} x\right)\right\}$ is a Cauchy sequence 
in $B$ for all $x \in A$. Since $B$ is complete, the sequence $\left\{\frac{1}{2^{n}} f\left(2^{n} x\right)\right\}$ converges in $B$ for all $x \in A$. So one can define the mapping $H: A \rightarrow B$ by

$$
H(x):=\lim _{n \rightarrow \infty} \frac{1}{2^{n}} f\left(2^{n} x\right)=\lim _{n \rightarrow \infty} \frac{1}{2^{n}} f_{i}\left(2^{n} x\right) \quad(i=0,1,2)
$$

for all $x \in A$. Letting $m=0$ and passing the limit when $n \rightarrow \infty$ in (4.13), we get (4.6). It follows from (4.1), (4.3) and (4.14) that

$$
\begin{aligned}
& \|H(\mu x+\mu y)-\mu H(x)-\mu H(y)\|_{B} \\
= & \lim _{n \rightarrow \infty} \frac{1}{2^{n}}\left\|f\left(2^{n} \mu x+2^{n} \mu y\right)-\mu f_{0}\left(2^{n} x\right)-\mu f_{1}\left(2^{n} y\right)\right\|_{B} \\
\leq & \lim _{n \rightarrow \infty} \frac{1}{2^{n}} \varphi\left(2^{n} x, 2^{n} y, 0\right)=0
\end{aligned}
$$

for all $\mu \in \mathbb{T}^{1}$ and all $x, y \in A$. Hence

$$
H(\mu x+\mu y)=\mu H(x)+\mu H(y)
$$

for all $\mu \in \mathbb{T}^{1}$ and all $x, y \in A$. By the same reasoning as in the proof of Theorem 2.1 of [11], the mapping $H: A \rightarrow B$ is $\mathbb{C}$-linear. It follows from (4.4) that the sequence $\left\{\frac{1}{2^{n}} f\left(2^{n} w\right)\right\}$ is a Cauchy sequence in $B_{0}$ for all $w \in A_{0}$. So $H(w) \in B_{0}$ for all $w \in A_{0}$. It follows from (4.1), (4.5) and (4.14) that

$$
\begin{aligned}
& \left\|H\left(\left\{w_{0}, x, w_{2}\right\}\right)-\left\{H\left(w_{0}\right), H(x), H\left(w_{2}\right)\right\}\right\|_{B} \\
\leq & \lim _{n \rightarrow \infty} \frac{1}{8^{n}}\left\|f\left(8^{n}\left\{w_{0}, x, w_{2}\right\}\right)-\left\{f_{0}\left(2^{n} w_{0}\right), f_{1}\left(2^{n} x\right), f_{2}\left(2^{n} w_{2}\right)\right\}\right\|_{B} \\
\leq & \lim _{n \rightarrow \infty} \frac{1}{8^{n}} \varphi\left(2^{n} w_{0}, 2^{n} x, 2^{n} w_{2}\right)=0
\end{aligned}
$$

for all $w_{0}, w_{2} \in A_{0}$ and all $x \in A$. Hence

$$
H\left(\left\{w_{0}, x, w_{1}\right\}\right)=\left\{H\left(w_{0}\right), H(x), H\left(w_{2}\right)\right\}
$$

for all $w_{0}, w_{2} \in A_{0}$ and all $x \in A$. So $H: A \rightarrow B$ is a proper $J C Q^{*}$-triple homomorphism. Now, we show that $H$ is unique. Let $T: A \rightarrow B$ be another proper $J C Q^{*}$-triple homomorphism satisfying (4.6). It follows from (4.2), (4.6) and (4.14) that

$$
\begin{aligned}
\|H(x)-T(x)\|_{B} & =\lim _{n \rightarrow \infty} \frac{1}{2^{n}}\left\|f\left(2^{n} x\right)-T\left(2^{n} x\right)\right\|_{B} \\
& \leq \frac{1}{2} \lim _{n \rightarrow \infty} \frac{1}{2^{n}} \widetilde{\varphi}\left(2^{n} x\right)=0
\end{aligned}
$$

for all $x \in A$. So $H=T$.

Theorem 4.2. Let $\phi: A \times A \times A \rightarrow[0,+\infty)$ be a function such that

$$
\begin{gathered}
\lim _{n \rightarrow \infty} 8^{n} \phi\left(\frac{x}{2^{n}}, \frac{y}{2^{n}}, \frac{z}{2^{n}}\right)=0, \\
\widetilde{\phi}(x):=\sum_{i=1}^{\infty} 2^{i}\left[\phi\left(\frac{x}{2^{i}}, \frac{x}{2^{i}}, 0\right)+\phi\left(\frac{x}{2^{i}}, 0,0\right)+\phi\left(0, \frac{x}{2^{i}}, 0\right)\right]<\infty
\end{gathered}
$$


for all $x, y, z \in A$. Suppose that $f, f_{i}: A \rightarrow B(0 \leq i \leq 2)$ are mappings satisfying $f(0)=f_{i}(0)=0$ and $f(w), f_{i}(w) \in B_{0}$ for all $w \in A_{0}$ and all $0 \leq i \leq 2$. Let

$$
\begin{gathered}
\left\|f(\mu x+\mu y+\mu z)-\mu f_{0}(x)-\mu f_{1}(y)-\mu f_{2}(z)\right\|_{B} \leq \phi(x, y, z), \\
\left\|f\left(w_{0}+w_{1}+w_{2}\right)-f_{0}\left(w_{0}\right)-f_{1}\left(w_{1}\right)-f_{2}\left(w_{2}\right)\right\|_{B_{0}} \leq \phi\left(w_{0}, w_{1}, w_{2}\right), \\
\left\|f\left(\left\{w_{0}, x, w_{1}\right\}\right)-\left\{f_{0}\left(w_{0}\right), f_{1}(x), f_{2}\left(w_{2}\right)\right\}\right\|_{B} \leq \phi\left(w_{0}, x, w_{2}\right)
\end{gathered}
$$

for all $\mu \in \mathbb{T}^{1}$, all $w_{0}, w_{1}, w_{2} \in A_{0}$ and all $x, y, z \in A$. Then there exists $a$ unique proper JCQ ${ }^{*}$-triple homomorphism $H: A \rightarrow B$ such that

$$
\begin{aligned}
\|f(x)-H(x)\|_{B} & \leq \frac{1}{2} \widetilde{\phi}(x), \\
\left\|f_{0}(x)-H(x)\right\|_{B} & \leq \frac{1}{2} \widetilde{\phi}(x)+\phi(x, 0,0), \\
\left\|f_{1}(x)-H(x)\right\|_{B} & \leq \frac{1}{2} \widetilde{\phi}(x)+\phi(0, x, 0), \\
\left\|f_{2}(x)-H(x)\right\|_{B} & \leq \frac{1}{2} \widetilde{\phi}(x)+\phi(0,0, x)
\end{aligned}
$$

for all $x \in A$.

Proof. Similar to Theorem 4.1, we get

$$
\|f(\mu x+\mu y+\mu z)-\mu f(x)-\mu f(y)-\mu f(z)\|_{B} \leq \Psi(x, y, z),
$$

where

$$
\Psi(x, y, z):=\phi(x, y, z)+\phi(x, 0,0)+\phi(0, y, 0)+\phi(0,0, z)
$$

for all $\mu \in \mathbb{T}^{1}$ and all $x, y, z \in A$. Letting $y=x, z=0$ and $\mu=1$ in (4.20), we get

$$
\|f(2 x)-2 f(x)\|_{B} \leq \Psi(x, x, 0)
$$

for all $x \in A$. Replacing $x$ by $\frac{x}{2^{n+1}}$ in (4.21) and multiplying both sides of (4.21) to $2^{n}$, we get

$$
\left\|2^{n+1} f\left(\frac{x}{2^{n+1}}\right)-2^{n} f\left(\frac{x}{2^{n}}\right)\right\|_{B} \leq 2^{n} \Psi\left(\frac{x}{2^{n+1}}, \frac{x}{2^{n+1}}, 0\right)
$$

for all $x \in A$ and all non-negative integers $n$. By (4.22), we get

$$
\left\|2^{n+1} f\left(\frac{x}{2^{n+1}}\right)-2^{m} f\left(\frac{x}{2^{m}}\right)\right\|_{B} \leq \sum_{i=m}^{n} 2^{i} \Psi\left(\frac{x}{2^{i+1}}, \frac{x}{2^{i+1}}, 0\right)
$$

for all $x \in A$ and all non-negative integers $n$ and $m$ with $n \geq m$. Thus we conclude from (4.16) and (4.23) that the sequence $\left\{2^{n} f\left(\frac{x}{2^{n}}\right)\right\}$ is a Cauchy sequence in $B$ for all $x \in A$. Since $B$ is complete, the sequence $\left\{2^{n} f\left(\frac{x}{2^{n}}\right)\right\}$ converges in $B$ for all $x \in A$. So one can define the mapping $H: A \rightarrow B$ by

$$
H(x):=\lim _{n \rightarrow \infty} 2^{n} f\left(\frac{x}{2^{n}}\right)=\lim _{n \rightarrow \infty} 2^{n} f_{i}\left(\frac{x}{2^{n}}\right) \quad(i=0,1,2)
$$


for all $x \in A$. Letting $m=0$ and passing the limit when $n \rightarrow \infty$ in (4.23), we get (4.19).

The rest of proof is similar to the proof of Theorem 4.1.

Corollary 4.3. Let $\theta, r_{i}(0 \leq i \leq 2)$ be non-negative real numbers such that $0<r_{i}<1$ (respectively, $r_{i}>3$ ) for all $0 \leq i \leq 2$. Suppose that $f, f_{i}: A \rightarrow B$ $(0 \leq i \leq 2)$ are mappings with $f(0)=f_{i}(0)=0$ and $f(w), f_{i}(w) \in B_{0}$ for all $w \in A_{0}$ and all $0 \leq i \leq 2$. Let

$$
\begin{aligned}
& \left\|f(\mu x+\mu y+\mu z)-\mu f_{0}(x)-\mu f_{1}(y)-\mu f_{2}(z)\right\|_{B} \\
\leq & \theta\left(\|x\|_{A}^{r_{0}}+\|y\|_{A}^{r_{1}}+\|z\|_{A}^{r_{2}}\right), \\
& \left\|f\left(w_{0}+w_{1}+w_{2}\right)-f_{0}\left(w_{0}\right)-f_{1}\left(w_{1}\right)-f_{2}\left(w_{2}\right)\right\|_{B_{0}} \\
\leq & \theta\left(\left\|w_{0}\right\|_{A}^{r_{0}}+\left\|w_{1}\right\|_{A}^{r_{1}}+\left\|w_{2}\right\|_{A}^{r_{2}}\right), \\
& \left\|f\left(\left\{w_{0}, x, w_{1}\right\}\right)-\left\{f_{0}\left(w_{0}\right), f_{1}(x), f_{2}\left(w_{2}\right)\right\}\right\|_{B} \\
\leq & \theta\left(\left\|w_{0}\right\|_{A}^{r_{0}}+\|x\|_{A}^{r_{1}}+\left\|w_{2}\right\|_{A}^{r_{2}}\right)
\end{aligned}
$$

for all $\mu \in \mathbb{T}^{1}$, all $w_{0}, w_{1}, w_{2} \in A_{0}$ and all $x, y, z \in A$. Then there exists a unique proper $J C Q^{*}$-triple homomorphism $H: A \rightarrow B$ such that

$$
\begin{aligned}
& \|f(x)-H(x)\|_{B} \leq 2 \theta\left[\frac{\|x\|_{A}^{r_{0}}}{\left|2-2^{r_{0}}\right|}+\frac{\|x\|_{A}^{r_{1}}}{\mid 2-2^{r_{1} \mid}}\right], \\
& \left\|f_{i}(x)-H(x)\right\|_{B} \leq 2 \theta\left[\frac{\|x\|_{A}^{r_{0}}}{\left|2-2^{r_{0}}\right|}+\frac{\|x\|_{A}^{r_{1}}}{\mid 2-2^{r_{1} \mid}}\right]+\theta\|x\|_{A}^{r_{i}}
\end{aligned}
$$

for all $x \in A$ and all $0 \leq i \leq 2$.

Theorem 4.4. Let $\theta, r_{i}(0 \leq i \leq 2)$ be non-negative real numbers such that $r_{0}+r_{1}+r_{2}<3$ and $0<r_{i}<1$ for some $0 \leq i \leq 2$. Suppose that $f, f_{i}: A \rightarrow B$ $(0 \leq i \leq 2)$ are mappings with $f(0)=0$ and $f(w), f_{0}(w), f_{2}(w) \in B_{0}$ for all $w \in A_{0}$. Let

$$
\left\|f(\mu x+\mu y+\mu z)-\mu f_{0}(x)-\mu f_{1}(y)-\mu f_{2}(z)\right\|_{B} \leq \theta\|x\|_{A}^{r_{0}}\|y\|_{A}^{r_{1}}\|z\|_{A}^{r_{2}},
$$
for all $\mu \in \mathbb{T}^{1}$, all $w_{0}, w_{2} \in A_{0}$ and all $x, y, z \in A$ (by putting $\|\cdot\|_{A}^{0}=1$ ). Then the mapping $f: A \rightarrow B$ is a proper JCQ*-triple homomorphism. Moreover, if $r_{i}, r_{j}>0$ for some $0 \leq i<j \leq 2$, then

$$
f(x)=f_{0}(x)-f_{0}(0)=f_{1}(x)-f_{1}(0)=f_{2}(x)-f_{2}(0)
$$

for all $x \in A$.

Proof. Without loss of generality, we may assume that $0<r_{2}<1$. It is clear that $f_{0}(0)+f_{1}(0)+f_{2}(0)=0$. By letting $y=z=0$ and $\mu=1$ in (4.24), we get

$$
f(x)=f_{0}(x)-f_{0}(0)
$$

for all $x \in A$. Similarly, we have

$$
f(x)=f_{1}(x)-f_{1}(0)
$$


for all $x \in A$. We have two cases:

Case I. $r_{0}=r_{1}=0$. We infer from (4.24) that

$$
\begin{aligned}
& \|f(\mu x+\mu y+\mu z)-\mu f(x)-\mu f(y)-\mu f(z)\|_{B} \\
\leq & \left\|f(\mu x+\mu y+\mu z)-\mu f_{0}(x)-\mu f_{1}(y)-\mu f_{2}(z)\right\|_{B} \\
& +\left\|f(z)-f_{0}(0)-f_{1}(0)-f_{2}(z)\right\|_{B} \leq 2 \theta\|z\|_{A}^{r_{2}}
\end{aligned}
$$

for all $\mu \in \mathbb{T}^{1}$ and all $x, y, z \in A$. By letting $z=0$ in (4.28), we get

$$
f(\mu x+\mu y)=\mu f(x)+\mu f(y)
$$

for all $\mu \in \mathbb{T}^{1}$ and all $x, y \in A$. By the same reasoning as in the proof of Theorem 2.1 of [11], the mapping $f: A \rightarrow B$ is $\mathbb{C}$-linear. It follows from (4.24), (4.26), and (4.27) that

$$
f(x)=\lim _{n \rightarrow \infty} \frac{1}{2^{n}} f_{0}\left(2^{n} x\right)=\lim _{n \rightarrow \infty} \frac{1}{2^{n}} f_{1}\left(2^{n} x\right)=\lim _{n \rightarrow \infty} \frac{1}{2^{n}} f_{2}\left(2^{n} x\right)
$$

for all $x \in A$. So (4.25) implies that

$$
\begin{aligned}
& \left\|f\left(\left\{w_{0}, x, w_{2}\right\}\right)-\left\{f\left(w_{0}\right), f(x), f\left(w_{2}\right)\right\}\right\|_{B} \\
= & \lim _{n \rightarrow \infty} \frac{1}{8^{n}}\left\|f\left(\left\{2^{n} w_{0}, 2^{n} x, 2^{n} w_{2}\right\}\right)-\left\{f_{0}\left(2^{n} w_{0}\right), f_{1}\left(2^{n} x\right), f_{2}\left(2^{n} w_{2}\right)\right\}\right\|_{B} \\
\leq & \theta \lim _{n \rightarrow \infty} \frac{2^{n r_{2}}}{8^{n}}\left\|w_{2}\right\|_{A}^{r_{2}}=0
\end{aligned}
$$

for all $w_{0}, w_{2} \in A_{0}$ and all $x \in A$. Therefore,

$$
f\left(\left\{w_{0}, x, w_{1}\right\}\right)=\left\{f\left(w_{0}\right), f(x), f\left(w_{2}\right)\right\}
$$

for all $w_{0}, w_{2} \in A_{0}$ and $x \in A$. So the mapping $f: A \rightarrow B$ is a proper $J C Q^{*}$-triple homomorphism.

Case II. $r_{0}>0$ or $r_{1}>0$. Without loss of generality, we may assume that $r_{1}>0$. Letting $x=y=0$ and $\mu=1$ in (4.24), we get that $f(z)=f_{2}(z)-f_{2}(0)$ for all $z \in A$. It follows from (4.24) that

$$
\begin{aligned}
& \|f(\mu x+\mu y+\mu z)-\mu f(x)-\mu f(y)-\mu f(z)\|_{B} \\
= & \left\|f(\mu x+\mu y+\mu z)-\mu f_{0}(x)-\mu f_{1}(y)-\mu f_{2}(z)\right\|_{B} \leq \theta\|x\|_{A}^{r_{0}}\|y\|_{A}^{r_{1}}\|z\|_{A}^{r_{2}}
\end{aligned}
$$

for all $\mu \in \mathbb{T}^{1}$ and all $x, y, z \in A$. By putting $z=0$ in the last inequality, we infer that the mapping $f$ is $\mathbb{C}$-linear. The rest of the proof is similar to the proof of Case I.

The following theorem is an alternative result of Theorem 4.4 and its proof is similar to the proof of Theorem 4.4.

Theorem 4.5. Let $\theta, r_{i}(0 \leq i \leq 2)$ be non-negative real numbers such that $r_{i}>3$ for some $0 \leq i \leq 2$. Suppose that $f, f_{i}: A \rightarrow B(0 \leq i \leq 2)$ are mappings satisfying (4.24) and (4.25) (by putting $\|\cdot\|_{A}^{0}=1$ ) with $f(0)=f_{i}(0)=0$ and $f(w), f_{0}(w), f_{2}(w) \in B_{0}$ for all $w \in A_{0}$. Then the mapping $f: A \rightarrow B$ 
is a proper JCQ $Q^{*}$-triple homomorphism. Moreover, if $r_{i}, r_{j}>0$ for some $0 \leq i<j \leq 2$, then

for all $x \in A$ and all $0 \leq i \leq 2$.

$$
f(x)=f_{i}(x)
$$

For $r_{0}=r_{1}=r_{2}=0$, we have the following theorem.

Theorem 4.6. Let $\theta$ be non-negative real number and let $f, f_{i}: A \rightarrow B(0 \leq$ $i \leq 2)$ be mappings such that $f(w), f_{i}(w) \in B_{0}(0 \leq i \leq 2)$ for all $w \in A_{0}$ and

$$
\begin{gathered}
\left\|f(\mu x+\mu y+\mu z)-\mu f_{0}(x)-\mu f_{1}(y)-\mu f_{2}(z)\right\|_{B} \leq \theta, \\
\left\|f\left(w_{0}+w_{1}+w_{2}\right)-f_{0}\left(w_{0}\right)-f_{1}\left(w_{1}\right)-f_{2}\left(w_{2}\right)\right\|_{B_{0}} \leq \theta, \\
\left\|f\left(\left\{w_{0}, x, w_{1}\right\}\right)-\left\{f_{0}\left(w_{0}\right), f_{1}(x), f_{2}\left(w_{2}\right)\right\}\right\|_{B} \leq \theta
\end{gathered}
$$

for all $\mu \in \mathbb{T}^{1}$, all $w_{0}, w_{1}, w_{2} \in A_{0}$ and all $x, y, z \in A$. Then there exists a unique proper $J C Q^{*}$-triple homomorphism $H: A \rightarrow B$ such that

$$
\begin{aligned}
\|f(x)+f(0)-H(x)\|_{B} & \leq 4 \theta+2 M, \\
\left\|f_{i}(x)-f_{i}(0)-H(x)\right\|_{B} & \leq 6 \theta+4 M \quad(i=0,1,2)
\end{aligned}
$$

for all $x \in A$, where $M=\left\|f_{0}(0)+f_{1}(0)+f_{2}(0)\right\|_{B}$.

Proof. Similar to the proof of Theorem 4.1, we have

$$
\|f(\mu x+\mu y+\mu z)-\mu f(x)-\mu f(y)-\mu f(z)\|_{B} \leq 4 \theta+2 M
$$

for all $x, y, z \in A$ and all $\mu \in \mathbb{T}^{1}$, where $M=\left\|f_{0}(0)+f_{1}(0)+f_{2}(0)\right\|_{B}$. Using the same proof as in Theorem 4.1, we infer that

$$
\left\|\frac{1}{2^{n+1}} f\left(2^{n+1} x\right)-\frac{1}{2^{m}} f\left(2^{m} x\right)-\sum_{i=m}^{n} \frac{1}{2^{i+1}} f(0)\right\|_{B} \leq(2 \theta+M) \sum_{i=m}^{n} \frac{1}{2^{i}}
$$

for all $x \in A$ and all non-negative integers $n$ and $m$ with $n \geq m$. Thus we conclude from (4.29) that the sequence $\left\{\frac{1}{2^{n}} f\left(2^{n} x\right)\right\}$ is a Cauchy sequence in $B$ for all $x \in A$. Since $B$ is complete, the sequence $\left\{\frac{1}{2^{n}} f\left(2^{n} x\right)\right\}$ converges in $B$ for all $x \in A$. So one can define the mapping $H: A \rightarrow B$ by

$$
H(x):=\lim _{n \rightarrow \infty} \frac{1}{2^{n}} f\left(2^{n} x\right)=\lim _{n \rightarrow \infty} \frac{1}{2^{n}} f_{i}\left(2^{n} x\right) \quad(i=0,1,2)
$$

for all $x \in A$.

The rest of the proof is similar to the proof of Theorem 4.1.

Theorem 4.7. Let $\theta \geq 0, r_{0}, r_{1}, r_{2}$ be real numbers such that $r_{0}+r_{1}>0$ and $r_{2}<0$. Assume that $f, f_{i}: A \rightarrow B(0 \leq i \leq 2)$ are mappings with $f_{0}(0)=f_{1}(0)=0$ and $f(w), f_{0}(w), f_{2}(w) \in B_{0}$ for all $w \in A_{0}$ and satisfying

$$
\begin{gathered}
\left\|f(x+y+z)-f_{0}(x)-f_{1}(y)-f_{2}(z)\right\|_{B} \leq \theta\|x\|_{A}^{r_{0}}\|y\|_{A}^{r_{1}}\|z\|_{A}^{r_{2}}, \\
\left\|f\left(\left\{w_{0}, x, w_{2}\right\}\right)-\left\{f_{0}\left(w_{0}\right), f_{1}(x), f_{2}\left(w_{2}\right)\right\}\right\|_{B} \leq \theta\left\|w_{0}\right\|_{A}^{r_{0}}\|x\|_{A}^{r_{1}}\left\|w_{2}\right\|_{A}^{r_{2}}
\end{gathered}
$$

for all $w_{2} \in A_{0} \backslash\{0\}$, all $w_{0} \in A_{0}\left(w_{0} \in A_{0} \backslash\{0\}\right.$ if $\left.r_{0}<0\right)$ and all $x, y \in$ $A\left(x \in A \backslash\{0\}\right.$ if $r_{0}<0$ and $y \in A \backslash\{0\}$ if $\left.r_{1}<0\right), z \in A \backslash\{0\}$. If the 
mappings $t \rightarrow f(t x)$ and $t \rightarrow f_{i}(t x)(0 \leq i \leq 2)$ are continuous in $0 \in \mathbb{R}$ for each fixed $x \in A$, then

(i) $f=f_{0}=f_{1}=f_{2}$,

(ii) the mapping $f: A \rightarrow B$ is a proper JCQ*-triple homomorphism.

Proof. Without loss of generality, we may assume that $r_{1}>0$. Letting $y=0$ in (4.30) and $x=0$ in (4.31), we get

$$
f(0)=0, \quad f(x+z)=f_{0}(x)+f_{2}(z), \quad(x, z \neq 0) .
$$

Replacing $x$ and $z$ by $\frac{x}{n}$ and $\frac{z}{n}$, respectively, in (4.32) and letting $n \rightarrow \infty$, we get that $f_{2}(0)=0$. Letting $y=-x$ in (4.30) and using (4.32), we get

$$
\left\|f(z)-f(x+z)-f_{1}(-x)\right\|_{B} \leq \theta\|x\|_{A}^{r_{0}+r_{1}}\|z\|_{A}^{r_{2}}, \quad(x, z \neq 0) .
$$

Therefore

$$
\lim _{n \rightarrow \infty} f\left(\frac{x}{n}+z\right)=f(z), \quad(x, z \neq 0) .
$$

Since $f(0)=0,(4.34)$ holds for all $x, z \in A$. It follows from (4.32) and (4.34) that $f=f_{2}$. So by replacing $z$ by $\frac{z}{n}$ in (4.32) and letting $n \rightarrow \infty$ and using (4.34), we get $f=f_{0}$. Hence (4.32) implies that the mapping $f$ is additive. Thus (4.30) means that

$$
\left\|f(y)-f_{1}(y)\right\|_{B} \leq \theta\|x\|_{A}^{r_{0}}\|y\|_{A}^{r_{1}}\|z\|_{A}^{r_{2}}, \quad(y \in A, x, z \neq 0) .
$$

So $f=f_{1}$, and this proves (i).

To prove (ii), since the mapping $f$ is additive, by the same reasoning as in the proof of the main result of [13], the mapping $f: A \rightarrow B$ is $\mathbb{C}$-linear. Now, (4.31) implies that

$$
\begin{aligned}
& \left\|f\left(\left\{w_{0}, x, w_{2}\right\}\right)-\left\{f\left(w_{0}\right), f(x), f\left(w_{2}\right)\right\}\right\|_{B} \\
= & \lim _{n \rightarrow \infty} \frac{1}{n}\left\|f\left(\left\{w_{0}, x, n w_{2}\right\}\right)-\left\{f\left(w_{0}\right), f(x), f\left(n w_{2}\right)\right\}\right\|_{B} \\
\leq & \theta \lim _{n \rightarrow \infty} n^{r_{2}-1}\left\|w_{0}\right\|_{A}^{r_{0}}\|x\|_{A}^{r_{1}}\left\|w_{2}\right\|_{A}^{r_{2}}=0
\end{aligned}
$$

for all $x \in A$ and all $w_{0}, w_{2} \in A_{0} \backslash\{0\}$. Since $f(0)=0$, therefore

$$
f\left(\left\{w_{0}, x, w_{2}\right\}\right)=\left\{f\left(w_{0}\right), f(x), f\left(w_{2}\right)\right\}
$$

for all $x \in A$ and all $w_{0}, w_{2} \in A_{0}$. So the mapping $f: A \rightarrow B$ is a proper $J C Q^{*}$-triple homomorphism.

Theorem 4.8. Let $\theta \geq 0$ and $r_{0}, r_{1}<0$ be real numbers and let $s_{0}, s_{1}, s_{2}$ be real numbers such that $s_{j} \neq 1$ for some $0 \leq j \leq 2$. Assume that $f: A \rightarrow B$ is a mapping with $f(0)=0$ and $f(w) \in B_{0}$ for all $w \in A_{0}$ and

$$
\|f(x+y)-f(x)-f(y)\|_{B} \leq \theta\|x\|_{A}^{r_{0}}\|y\|_{A}^{r_{1}},
$$

$$
\left\|f\left(\left\{w_{0}, x, w_{1}\right\}\right)-\left\{f\left(w_{0}\right), f(x), f\left(w_{2}\right)\right\}\right\|_{B} \leq \theta\left\|w_{0}\right\|_{A}^{s_{0}}\|x\|_{A}^{s_{1}}\left\|w_{2}\right\|_{A}^{s_{2}}
$$


for all $w_{0}, w_{2} \in A_{0} \backslash\{0\}$ and all $x, y \in A \backslash\{0\}$. If the mapping $t \rightarrow f(t x)$ is continuous in $0 \in \mathbb{R}$ for each fixed $x \in A$, then the mapping $f: A \rightarrow B$ is a proper JCQ*-triple homomorphism.

Proof. Let $y \in A \backslash\{0\}$. Replacing $x$ and $y$ in (4.35) by $\frac{y}{2}+n y$ and $\frac{y}{2}-n y$, respectively, we get

$$
f(y)=\lim _{n \rightarrow \infty}\left[f\left(\frac{y}{2}+n y\right)+f\left(\frac{y}{2}-n y\right)\right]
$$

for all $y \in A \backslash\{0\}$. Since $f(0)=0,(4.37)$ holds for all $y \in A$. Let $x, y \in A \backslash\{0\}$. It follows from (4.35) and (4.37) that

$$
\begin{aligned}
& \|f(x+y)-f(x)-f(y)\|_{B} \\
= & \lim _{n \rightarrow \infty} \| f\left(\frac{x+y}{2}+n(x+y)\right)+f\left(\frac{x+y}{2}-n(x+y)\right) \\
& -f\left(\frac{x}{2}+n x\right)-f\left(\frac{x}{2}-n x\right)-f\left(\frac{y}{2}+n y\right)-f\left(\frac{y}{2}-n y\right) \|_{B} \\
\leq & \limsup _{n \rightarrow \infty}\left\|f\left(\frac{x+y}{2}+n(x+y)\right)-f\left(\frac{x}{2}+n x\right)-f\left(\frac{y}{2}+n y\right)\right\|_{B} \\
& +\limsup _{n \rightarrow \infty}\left\|f\left(\frac{x+y}{2}-n(x+y)\right)-f\left(\frac{x}{2}-n x\right)-f\left(\frac{y}{2}-n y\right)\right\|_{B} \\
\leq & \theta\left[\lim _{n \rightarrow \infty}\left(\frac{1}{2}+n\right)^{r_{0}+r_{1}}+\lim _{n \rightarrow \infty}\left(n-\frac{1}{2}\right)^{r_{0}+r_{1}}\right]\|x\|_{A}^{r_{0}}\|y\|_{A}^{r_{1}}=0 .
\end{aligned}
$$

So we have $f(x+y)=f(x)+f(y)$ for all $x, y \in A \backslash\{0\}$. Since $f(0)=0$, we get that the mapping $f$ is additive. By the same reasoning as in the proof of the main result of [13], the mapping $f: A \rightarrow B$ is $\mathbb{C}$-linear. Without loss of generality we may assume that $s_{0} \neq 1$. Let $s_{0}<1$ (we have similar proof when $\left.s_{0}>1\right)$. It follows from (4.36) that

$$
\begin{aligned}
& \left\|f\left(\left\{w_{0}, x, w_{2}\right\}\right)-\left\{f\left(w_{0}\right), f(x), f\left(w_{2}\right)\right\}\right\|_{B} \\
= & \lim _{n \rightarrow \infty} \frac{1}{n}\left\|f\left(\left\{n w_{0}, x, w_{2}\right\}\right)-\left\{f\left(n w_{0}\right), f(x), f\left(w_{2}\right)\right\}\right\|_{B} \\
\leq & \theta \lim _{n \rightarrow \infty} n^{s_{0}-1}\left\|w_{0}\right\|_{A}^{s_{0}}\|x\|_{A}^{s_{1}}\left\|w_{2}\right\|_{A}^{s_{2}}=0
\end{aligned}
$$

for all $x \in A \backslash\{0\}$ and all $w_{0}, w_{2} \in A_{0} \backslash\{0\}$. Since $f(0)=0$, we get that

$$
f\left(\left\{w_{0}, x, w_{2}\right\}\right)=\left\{f\left(w_{0}\right), f(x), f\left(w_{2}\right)\right\}
$$

for all $x \in A$ and all $w_{0}, w_{2} \in A_{0}$. So the mapping $f: A \rightarrow B$ is a proper $J C Q^{*}$-triple homomorphism.

\section{References}

[1] J. P. Antoine, A. Inoue, and C. Trapani, Partial ${ }^{*}$-algebras and Their Operator Realizations, Mathematics and its Applications, 553. Kluwer Academic Publishers, Dordrecht, 2002.

[2] F. Bagarello, A. Inoue, and C. Trapani, Some classes of topological quasi *-algebras, Proc. Amer. Math. Soc. 129 (2001), no. 10, 2973-2980 
[3] F. Bagarello and C. Trapani, States and representations of $C Q^{*}$-algebras, Ann. Inst. H. Poincare Phys. Theor. 61 (1994), no. 1, 103-133.

[4] —_ CQ*-algebras: structure properties, Publ. Res. Inst. Math. Sci. 32 (1996), no. 1, 85-116.

[5] - Morphisms of certain Banach $C^{*}$-modules, Publ. Res. Inst. Math. Sci. 36 (2000), no. 6, 681-705.

[6] P. Găvruta, A generalization of the Hyers-Ulam-Rassias stability of approximately additive mappings, J. Math. Anal. Appl. 184 (1994), no. 3, 431-436.

[7] D. H. Hyers, On the stability of the linear functional equation, Proc. Nat. Acad. Sci. U. S. A. 27 (1941), 222-224.

[8] G. Lassner, Topological algebras and their applications in quantum statistics, Wiss. Z. Karl-Marx-Univ. Leipzig Math.-Natur. Reihe 30 (1981), no. 6, 572-595.

[9] G. Lassner and G. A. Lassner, Qu*-algebras and twisted product, Publ. Res. Inst. Math. Sci. 25 (1989), no. 2, 279-299.

[10] A. Najati and C. Park, Hyers-Ulam-Rassias stability of homomorphisms in quasiBanach algebras associated to the Pexiderized Cauchy functional equation, J. Math. Anal. Appl. 335 (2007), no. 2, 763-778.

[11] C. Park, Homomorphisms between Poisson JC ${ }^{*}$-algebras, Bull. Braz. Math. Soc. (N.S.) 36 (2005), no. 1, 79-97.

[12] C. Park and Th. M. Rassias, Homomorphisms and derivations in proper JCQ*-triples, J. Math. Anal. Appl. 337 (2008), no. 2, 1404-1414.

[13] Th. M. Rassias, On the stability of the linear mapping in Banach spaces, Proc. Amer. Math. Soc. 72 (1978), no. 2, 297-300.

[14] S. M. Ulam, A Collection of Mathematical Problems, Interscience Tracts in Pure and Applied Mathematics, no. 8 Interscience Publishers, New York-London, 1960.

\author{
AbBas Najati \\ Department of Mathematics \\ FACULTY OF SCIENCES \\ UNIVERSITY OF MOHAGHEGH ARDABILI \\ ArDaBIL, Iran \\ E-mail address: a.najati@yahoo.com \\ G. ZAMANI ESKANDANI \\ FaCUlty of Mathematical Sciences \\ UNIVERSITY OF TABRIZ \\ TABRIZ, IRAN \\ E-mail address: zamani@tabrizu.ac.ir \\ CHOONKIL PARK \\ DEPARTMENT OF MATHEMATICS \\ HANYANG UNIVERSITY \\ SEOUL 133-791, KOREA \\ E-mail address: baak@hanyang.ac.kx
}

